\title{
The Expression of Tumor-Associated Macrophages in Papillary Thyroid Carcinoma
}

\author{
Bo Hyun Kim
}

Department of Internal Medicine and Biomedical Research Institute, Pusan National University School of Medicine, Busan, Korea

Solid tumors contain not only malignant cells, but also extracellular matrix and many other nonmalignant cells including fibroblasts, endothelial cells, and inflammatory cells such as macrophages, neutrophils, and lymphocytes. The presence of inflammatory cells within solid tumors suggests that the inflammatory microenvironment could play a major role in promoting tumorigenesis and progression. Macrophage infiltrates in the context of or surrounding a variety of malignancies represent the host's immune response to the tumor [1,2].

It was recently reported that tumor-associated macrophages (TAMs) had important roles in the tumor progression and metastasis of various cancers, including advanced thyroid cancer [3-9]. However, the role of TAMs in papillary thyroid carcinoma (PTC) has not been fully elucidated. Fiumara et al. [10] studied the tissue distribution and prognostic significance of TAMs in a retrospective series of 121 PTCs. They found tumor-infiltrating macrophages in approximately $70 \%$ of cases. Phagocytosis of neoplastic cells by macrophages was observed in approximately $15 \%$ of tumors, and none of these tumors developed distant metastases. These data suggest that neoplastic cell phagocytosis by macrophages and lymphocytic infiltration play a protective role in the development of distant metastases in patients with PTC.

Conversely, emerging data suggest that TAMs promote tumor progression and metastasis in PTC. Ryder et al. [8] reported that an increased density of TAMs was associated with tu-

Corresponding author: Bo Hyun Kim

Department of Internal Medicine and Biomedical Research Institute,

Pusan National University School of Medicine, 179 Gudeok-ro, Seo-gu,

Busan 602-739, Korea

Tel: +82-51-240-7678, Fax: +82-51-254-3237, E-mail: pons71@hanmail.net mor progression in advanced thyroid cancers and that there was a significant correlation between increased TAMs and histological grade, tumor invasiveness, and decreased cancerrelated survival in PTC. Very recently, they also reported that TAMs promoted PTC progression in BRAF-induced PTC mouse models, and that targeting CCR2-expressing cells during $B R A F$ induction reduced TAM density and impaired PTC development [11]. These results suggested that therapeutic strategies targeting TAMs may be beneficial in the treatment of advanced PTC.

Qing et al. [9] investigated TAMs density in both benign thyroid lesions and PTC tumors by CD68 immunostaining. They found that the overall density of TAMs was significantly higher in PTC tumors compared with thyroid goiter and follicular adenoma. In addition, the density of TAMs was positively associated with lymph node (LN) metastasis in TNM stages III/VI compared with stages I/II. However, no association was observed with other common tumor features, including $B R A F$ mutation.

In this issue, Kim et al. [12] investigated the expression of TAMs in 36 PTC patients with LN metastasis using immunohistochemical staining with anti-CD68 antibody. They reported that a higher density of TAMs was correlated with larger tumor size, suggesting a protumorigenic role of TAMs in PTCs. Comparing clinicopathologic characteristics among low $(<25 \%)$ and high (25\% to $70 \%)$ TAM density groups, primary tumor
Copyright $\odot 2013$ Korean Endocrine Society

This is an Open Access article distributed under the terms of the Creative Commons Attribution Non-Commercial License (http://creativecommons.org/ licenses/by-nc/3.0/) which permits unrestricted non-commercial use, distribution, and reproduction in any medium, provided the original work is properly cited. 
size was larger in the high density group compared to the low density group $(2.0 \pm 0.1$ vs. $1.5 \pm 0.1 ; P=0.009)$. However, there was no significant association between high TAM density and poorer clinicopathologic characteristics including multifocality, LN metastasis, and extrathyroidal extension. These data were not in agreement with those of previous studies, which reported that high TAM density was correlated with tumor invasiveness and poor clinicopathologic characteristics, excepting tumor size $[8,9,11]$.

Kim et al. [12] also analyzed the morphological characteristics of TAMs in PTC. They found that TAMs had thin, elongated cytoplasmic extensions, forming a canopy structure over tumor cells, and that the morphological characteristics of thyroid cancer tissue were well maintained irrespective of the presence of TAMs. In view of this, they inferred that TAMs do not play key role in tumor development in PTC. However, Caillou et al. [13] reported that anaplastic thyroid cancer displayed a very dense network of interconnected ramified TAMs in direct contact with intermingled cancer cells, and that this TAM network was directly related to the aggressiveness of thyroid cancer.

Unfortunately, Kim et al. [12] conducted this study using anti-CD68 antibody on only a small sample of PTCs with LN metastasis. A major limitation of this study was the lack of follow-up data with which to evaluate long-term outcomes. Therefore, further studies using a more specific antibody and larger sample sizes of various stages of PTC are warranted to investigate the role of TAMs in tumor development, tumor invasiveness, and metastasis of PTC. Finally, TAM-targeted pharmacologic therapy in patients with advanced thyroid cancer should also be investigated.

\section{CONFLICTS OF INTEREST}

No potential conflict of interest relevant to this article was reported.

\section{REFERENCES}

1. Balkwill F, Mantovani A. Inflammation and cancer: back to Virchow? Lancet 2001;357:539-45.

2. Coussens LM, Werb Z. Inflammation and cancer. Nature 2002;420:860-7.

3. Bingle L, Brown NJ, Lewis CE. The role of tumour-associated macrophages in tumour progression: implications for new anticancer therapies. J Pathol 2002;196:254-65.
4. Heusinkveld M, van der Burg SH. Identification and manipulation of tumor associated macrophages in human cancers. J Transl Med 2011;9:216.

5. Tsutsui S, Yasuda K, Suzuki K, Tahara K, Higashi H, Era S. Macrophage infiltration and its prognostic implications in breast cancer: the relationship with VEGF expression and microvessel density. Oncol Rep 2005;14:425-31.

6. Hirayama S, Ishii G, Nagai K, Ono S, Kojima M, Yamauchi C, Aokage K, Hishida T, Yoshida J, Suzuki K, Ochiai A. Prognostic impact of CD204-positive macrophages in lung squamous cell carcinoma: possible contribution of Cd204-positive macrophages to the tumor-promoting microenvironment. J Thorac Oncol 2012;7:1790-7.

7. Takayama H, Nishimura K, Tsujimura A, Nakai Y, Nakayama M, Aozasa K, Okuyama A, Nonomura N. Increased infiltration of tumor associated macrophages is associated with poor prognosis of bladder carcinoma in situ after intravesical bacillus Calmette-Guerin instillation. J Urol 2009; 181:1894-900.

8. Ryder M, Ghossein RA, Ricarte-Filho JC, Knauf JA, Fagin JA. Increased density of tumor-associated macrophages is associated with decreased survival in advanced thyroid cancer. Endocr Relat Cancer 2008;15:1069-74.

9. Qing W, Fang WY, Ye L, Shen LY, Zhang XF, Fei XC, Chen X, Wang WQ, Li XY, Xiao JC, Ning G. Density of tumorassociated macrophages correlates with lymph node metastasis in papillary thyroid carcinoma. Thyroid 2012;22: 905-10.

10. Fiumara A, Belfiore A, Russo G, Salomone E, Santonocito GM, Ippolito O, Vigneri R, Gangemi P. In situ evidence of neoplastic cell phagocytosis by macrophages in papillary thyroid cancer. J Clin Endocrinol Metab 1997;82:1615-20.

11. Ryder M, Gild M, Hohl TM, Pamer E, Knauf J, Ghossein R, Joyce JA, Fagin JA. Genetic and pharmacological targeting of CSF-1/CSF-1R inhibits tumor-associated macrophages and impairs BRAF-induced thyroid cancer progression. PLoS One 2013;8:e54302.

12. Kim S, Cho SW, Min HS, Kim KM, Yeom GJ, Kim EY, Lee KE, Yun YG, Park DJ, Park YJ. The expression of tumor-associated macrophages in papillary thyroid carcinoma. Endocrinol Metab 2013;28:192-8.

13. Caillou B, Talbot M, Weyemi U, Pioche-Durieu C, Al Ghuzlan A, Bidart JM, Chouaib S, Schlumberger M, Dupuy C. Tumor-associated macrophages (TAMs) form an interconnected cellular supportive network in anaplastic thyroid carcinoma. PLoS One 2011;6:e22567. 